University of South Carolina

Scholar Commons

8-1-2002

\title{
Low-Loss High Power RF Switching Using Multifinger AlGaN/GaN MOSHFETS
}

\author{
Alexei Koudymov \\ University of South Carolina - Columbia \\ Xuhong $\mathrm{Hu}$ \\ University of South Carolina - Columbia \\ Kirill Simin \\ University of South Carolina - Columbia \\ Grigory Simin \\ University of South Carolina - Columbia, simin@engr.sc.edu \\ Mohammod Ali \\ University of South Carolina - Columbia, alimo@engr.sc.edu
}

See next page for additional authors

Follow this and additional works at: https://scholarcommons.sc.edu/elct_facpub

Part of the Electrical and Computer Engineering Commons

Publication Info

Published in IEEE Electron Device Letters, Volume 23, 2002, pages 449-451.

http://ieeexplore.iee. $\mathrm{org} / \mathrm{xpl} /$ Recentlssue.jsp?punumber=55

(C) 2002 by IEEE

This Article is brought to you by the Electrical Engineering, Department of at Scholar Commons. It has been accepted for inclusion in Faculty Publications by an authorized administrator of Scholar Commons. For more information, please contact digres@mailbox.sc.edu. 
Author(s)

Alexei Koudymov, Xuhong Hu, Kirill Simin, Grigory Simin, Mohammod Ali, Jinwei Yang, and M. Asif Khan

This article is available at Scholar Commons: https://scholarcommons.sc.edu/elct_facpub/22 


\title{
Low-Loss High Power RF Switching Using Multifinger AlGaN/GaN MOSHFETs
}

\author{
Alexei Koudymov, Xuhong Hu, Kirill Simin, Grigory Simin, Member, IEEE, Mohammod Ali, Member, IEEE, \\ Jinwei Yang, and M. Asif Khan, Member, IEEE
}

\begin{abstract}
We demonstrate a novel RF switch based on a multifinger AlGaN/GaN MOSHFET. Record high saturation current and breakdown voltage, extremely low gate leakage current and low gate capacitance of the III-N MOSHFETs make them excellent active elements for RF switching. Using a single element test circuit with 1-mm wide multifinger MOSHFET we achieved 0.27 $\mathrm{dB}$ insertion loss and more than $\mathbf{4 0} \mathrm{dB}$ isolation. These parameters can be further improved by impedance matching and by using submicron gate devices. The maximum switching power extrapolated from the results for $1 \mathrm{~A} / \mathrm{mm} 100 \mu \mathrm{m}$ wide device exceeds $40 \mathrm{~W}$ for a $1-\mathrm{mm}$ wide $2-\mathrm{A} / \mathrm{mm}$ MOSHFET.
\end{abstract}

Index Terms-Field-effect transistor (FET), GaN, HEMT, heterostructure field-effect transistor (HFET), MOSHFET, RF, switch, wireless.

\section{INTRODUCTION}

$\mathbf{T}$ HE RAPID growth of wireless communications has resulted in the proliferation of a large variety of new wireless devices ranging from mobile phones and PDAs to GPS receivers. A transmitter/receiver (T/R) switch is one of the key elements of these devices as well as of many systems such as radars and phase arrays. RF switches are also essential for adding or reducing phase delay when used in conjunction with transmission line segments. Such phase delay or phase shifting is necessary for scanning antenna arrays. Low-loss RF switches are being sought for multifunction antenna systems where a single aperture is used to serve multiple applications simply by switching between individual radiating elements. Traditional $\mathrm{p}-\mathrm{i}-\mathrm{n}$ diode based $\mathrm{T} / \mathrm{R}$ switches encounter additional dc losses because the diode consumes power. This is why there has been quite a strong drive to design switches based on active devices [1]-[3], most of the research to date being done using GaAs devices [4], [5]

AlGaN/GaN heterostructure field-effect transistor (HFET) is one of the most promising new microwave devices, which has been a topic of intensive investigations since the first report in 1991 [6]. These devices exploit high sheet carrier density $\left(>1.5 \times 10^{13} \mathrm{~cm}^{-2}\right.$, an order of magnitude higher than that for GaAs/AlGaAs heterostructures) and high room temperature mobility ( $>2000 \mathrm{~cm}^{2} / \mathrm{V} \cdot \mathrm{s}$ ). These high sheet density and mobility of two-dimensional (2-D) electrons result in very low sheet channel resistance, 300-400 $\Omega$ /square and

Manuscript received April 24, 2002; revised May 17, 2002. This work was supported by SMDC Contract DASG60-00-10003, monitored by M. F. Clarke and T. Bauer, SMDC, and Dr. K. Wu, MDA. The review of this letter was arranged by Editor D. Ueda.

The authors are with the Department of Electrical Engineering, University of South Carolina, Columbia, SC 29208 USA (e-mail: simin@engr.sc.edu).

Publisher Item Identifier 10.1109/LED.2002.801301. record high saturation currents, in excess of $1 \mathrm{~A} / \mathrm{mm}$ [7]. Combining the advantages of the MOS structure (the low gate leakage current) and $\mathrm{AlGaN} / \mathrm{GaN}$ heterointerface (providing high-density high-mobility 2-DEG channel), we have recently demonstrated a novel GaN-AlGaN metal-oxide-semiconductor HFETs (MOSHFETs) [8]. The MOSHFETs posses extremely low gate currents, which allow them to operate at positive gate bias. Due to this, the maximum saturation current achievable in MOSHFETs almost doubles compared to regular HFET devices. Besides, the gate capacitance of MOSHFETs is lower due to larger gate-to-channel separation. The breakdown voltage of MOSHFETs is of the same value or higher as that of HFETs [9]. It can be as high as $500 \mathrm{~V}$ as shown in [9]. These features make MOSHFET an excellent candidate for high-power low-loss RF switches, phase shifters and attenuators. In this paper, we for the first time demonstrate the use of a large periphery multifinger (MF) MOSHFET as an active element for broadband high-power RF switching.

\section{MOSHFET RF SWITCH CHARACTERISTICS}

For this study, the MOSHFET devices fabricated and reported earlier [10] were used. The devices with the gate length of $1 \mu \mathrm{m}$ were fabricated over insulating $\mathrm{SiC}$ substrates. The device structure consisted of 7-nm-thick $\mathrm{SiO}_{2}$ layer, 25-nm- $\mathrm{Al}_{0.2} \mathrm{Ga}_{0.8} \mathrm{~N}$ barrier, and 1.5- $\mu \mathrm{m}$-thick undoped GaN buffer [see Fig. 1(a)]. In order to achieve high saturation currents large periphery MF devices were fabricated using eight gate fingers connected in parallel resulting in the total gate width of $1 \mathrm{~mm}$ [10]. Interconnections were made using the bridges with $\mathrm{SiO}_{2}$ isolation between source and gate metallization. The threshold voltage of the devices was around $V_{T}=-9 \mathrm{~V}$. The maximum saturation current of about $2 \mathrm{~A} / \mathrm{mm}$ was obtained at gate voltage $V_{G}=+5 \mathrm{~V}$ (measured in pulsed mode to avoid heating). The cutoff frequency of this large periphery MOSHFET was $f_{T} \sim 8.5 \mathrm{GHz}$.

For the RF switching study the MF MOSHFET was wirebonded into a $50 \Omega$ microstrip line as shown in Fig. 1(b). The transmission characteristics were measured using an HP 8510C vector network analyzer. The results for both the "ON" state $\left(V_{G} \approx+5 \mathrm{~V}\right)$ and the "OFF" state $\left(V_{G} \approx-10 \mathrm{~V}\right)$ are shown in Fig. 2. As seen, in the "ON" state the insertion loss is as low as $0.27 \mathrm{~dB}$ at $100 \mathrm{MHz}$ and increases gradually up to $0.5 \mathrm{~dB}$ at $1 \mathrm{GHz}$. In the "ON" state, the channel resistance $R_{C h}$ of the MOSHFET is low, which ensures low-loss input-output transmission. In this state, the drain-source capacitance is shunted by the $R_{C h}$, therefore, the transmission is almost frequency independent. The value of the $R_{C h}$ can be estimated from the device parameters as $R_{C h}=2 R_{C}+R_{G S}+R_{G D}+R_{G}\left(V_{G}\right)$, where 


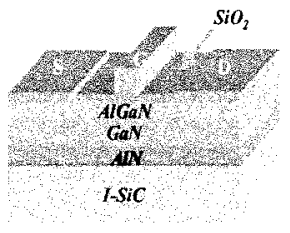

(a)

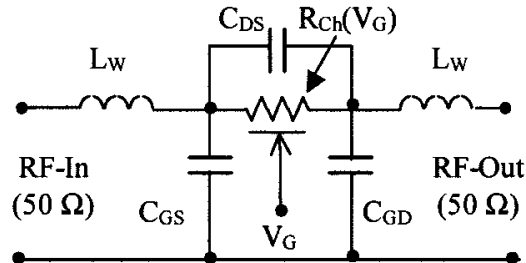

(b)
Fig. 1. (a) MOSHFET device structure and (b) schematic layout of the MOSHFET RF switch.

the $R_{G S}$ and $R_{G D}$ are the resistances of the source-gate and gate- drain openings, $R_{C}$ is the contact resistance and $R_{G}\left(V_{G}\right)$ is the voltage dependent resistance of the channel under the gate. At high positive gate bias, especially for the short gate devices, $R_{G}\left(V_{G}\right) \ll R_{C h}$. Also, $R_{G S}=R_{S H} \times L_{G S} / W$ and $R_{G D}=$ $R_{S H} \times L_{G D} / W$, where $R_{S H}=1 /\left(q N_{S} \mu_{n}\right)$ is the layer sheet resistance, $L_{G S}$ and $L_{G D}$ are the source-gate and gate-drain spacing. For the MOSHFET used in our experiments, $R_{S H} \approx$ $400 \Omega, L_{G S} \approx L_{G D} \approx 1.5 \mu \mathrm{m}$, and the contact resistance $R_{C} \approx 1 \Omega / \mathrm{mm}$ [6], [10], which results in $R_{C h} \approx 3 \Omega / \mathrm{mm}$. The insertion loss of the RF switch connected into a transmission line with the characteristic impedance $Z_{0}\left(Z_{0}=50 \Omega\right.$ in our experiment), assuming $R_{O N} \ll Z_{0}$ can be estimated as $L_{I n s}(\mathrm{~dB})=-20 \log \left(1 /\left(1+R_{O N} / 2 Z_{0}\right)\right) \approx 0.087 R_{O N}$. It follows that for $R_{O N}=R_{C h} \approx 3 \Omega$ for the 1-mm-wide MOSHFET used in the experiment $L_{\text {Ins }} \approx 0.26 \mathrm{~dB}$, which agrees perfectly with the experimental data. The frequency dependence of the switch transmission characteristic is determined by the source-gate and gate-drain capacitances and the inductances of the wirings. The dashed lines in the Fig. 2 show the results of simulations, which include all the device parameters and wiring inductances shown in the equivalent circuit of Fig. 1(b). The wiring was made using $20-\mu \mathrm{m}$ diameter gold wires with about $2 \mathrm{~mm}$ total length. The wiring inductance was estimated to be $L_{w} \approx 2 \mathrm{nH}$. As seen, these results agree very well with the experimental data. According to the simulations, the wiring inductances play the most important role in the measured frequency dependence. Note no special matching circuits were used in these experiments. The frequency bandwidth of the MOSHFET switch can be significantly expanded by impedance matching.

In the "OFF" state, the gate bias at the MOSHFET is below the threshold voltage and the pinchoff channel current is as low as several nanoamperes. Correspondingly, the channel resistance increases and the switch transmission is determined by the source-drain capacitance. In this case, the isolation can be estimated as $L_{I s}(\mathrm{~dB})=20 \log \left(1 /\left(\left|1+Z_{D S} / 2 Z_{0}\right|\right)\right)$, where $Z_{D S}=-1\left(j \omega C_{D S}\right)$ is the impedance of the MOSHFET at the subthreshold gate bias conditions. The source-to-drain capacitance of the MOSHFET at the subthreshold gate bias was measured using HP4284A L-C-R meter at $1 \mathrm{MHz}$. The measured value of $0.24 \mathrm{pF} / \mathrm{mm}$ agrees well with $C_{D S} \approx 0.15 \mathrm{pF} / \mathrm{mm}$ estimated from the planar electrode capacitance, provided that an additional capacitance of the planar probe contacts of around $0.1 \mathrm{pF} / \mathrm{mm}$ is taken into account. We used the following expression [11] for capacitance estimation: $C_{0}=\varepsilon_{0}\left(1+\varepsilon_{s}\right)\left(K(k) / K\left(k^{\prime}\right)\right)$, where $k=\tan ^{2}\left(\pi W_{P} / 4\left(L_{D S}+W_{P}\right)\right)$ and $k^{\prime}=\left(1-k^{2}\right)^{1 / 2}, K$

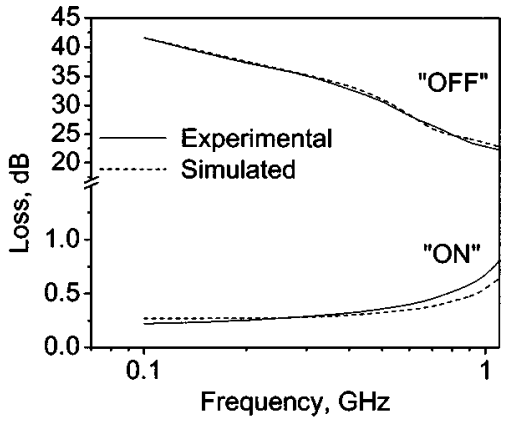

Fig. 2. Frequency dependencies of the MOSHFET switch insertion loss and isolation. Solid lines are experimental data and dashed lines are simulated.

is the elliptic integral of the first kind, $W_{P}=50 \mu \mathrm{m}$ is the contact pad length, and $L_{D S}=5 \mu \mathrm{m}$ is the electrode spacing.

The simulated results of MOSHFET switch isolation, shown by the dashed lines in the Fig. 2, agree well with the experiment.

The maximum switching power is one of the most important parameters of the RF switch. The fundamental advantage of the III-N based MOSHFET switch for high power applications is the unique combination of very high saturation current and high breakdown voltage. For experimental study of the maximum switching power, we have used the HP8314B RF Generator in combination with the HP83020A power amplifier. The maximum output power was around $30 \mathrm{dBm}$ at $2 \mathrm{GHz}$. As we will show in thew following, the saturation power of the 1-mm-wide MOSHFET switch is well above this level $(1 \mathrm{~W})$. Therefore, in order to measure the maximum switching power, we used the MOSHFET with the total gate width of $0.1 \mathrm{~mm}$ and relatively low, about $1 \mathrm{~A} / \mathrm{mm}$ at $V_{G}=+5 \mathrm{~V}$, saturation current. The experimental data is presented in Fig. 3. As the CW input signal increases, the output power increases linearly up to $24 \mathrm{dBm}(250 \mathrm{~mW})$ with the insertion loss of $4.5 \mathrm{~dB}$ at $2 \mathrm{~dB}$ compression. The results of this experiment can be used to evaluate the maximum switching power of the large periphery MF MOSHFET. The maximum power $P_{M O N}$ that can be switched by the active element in the "ON" state can be expressed as $P_{M O N}=P_{m}\left(1 / 1-K_{p}\right)$, where $P_{m}=V_{P S} I_{P S} / 2$ is the maximum power dissipated by the active element, $V_{P S}$ and $I_{P S}$ are the device voltage and current amplitudes at the saturation, and $K_{p}$ is power transmission coefficient of the switch $\left(K_{p}=10^{\left(L_{I n s} / 10\right)}\right)$. The saturation current of MOSHFET, per unit device width $I_{S M}=q N_{s} v_{s}$, where $N_{s}$ is the gate bias dependent electron sheet density, $v_{s}$ is the electron velocity in the channel. As estimated in [6], $v_{s} \approx 5 \times 10^{6} \mathrm{~cm} / \mathrm{s}$. The maximum electron sheet density in the MOSHFET channel at high positive gate bias, $N_{s h} \approx 2.5 \times 10^{13} \mathrm{~cm}^{-2}$ [7], which results in the maximum current of $I_{S M} \approx 2 \mathrm{~A} / \mathrm{mm}$. The knee voltage $V_{K n} \approx I_{S M}\left(2 R_{C}+R_{G S}+R_{G D}\right)+E_{S} L_{G}$, where $E_{S}$ is the characteristic field for the 2-D electron velocity saturation, and $L_{G}$ is the gate length. At high gate bias, the voltage drop across the $R_{C}, R_{G S}$, and $R_{G D}$ dominates in the expression for $V_{K n}$ compared to the $\left(E_{S} L_{G}\right)$ term, especially for the short-gate devices. Therefore, $V_{K n} \approx I_{D S} R_{C h} \approx 6 \mathrm{~V}$ for the typical MOSHFET parameters described previously in this paper. The experimental value of $V_{K n} \approx 6.5 \mathrm{~V}$ at $V_{G}=+5 \mathrm{~V}$ agrees very well with the previous estimation. For the $0.1-\mathrm{mm}$-wide, $1-\mathrm{A} / \mathrm{mm}$ device used in the power saturation experiment, at 24- $\mathrm{dBm}$ input power and 4-dB loss, the power dissipated in the 


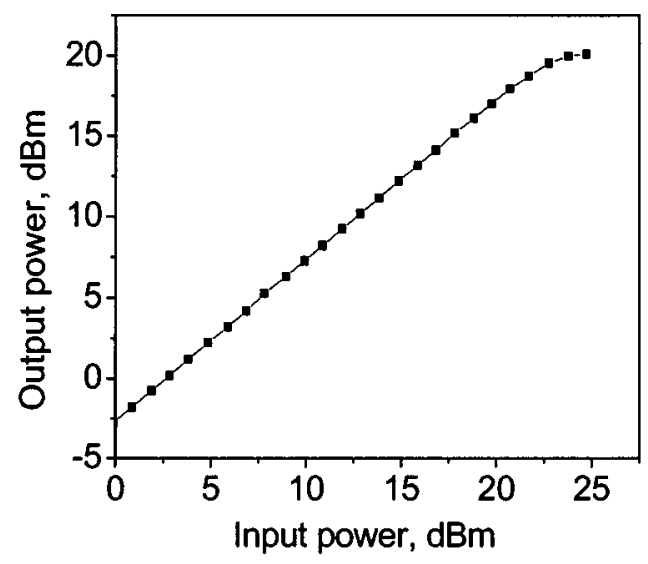

(a)

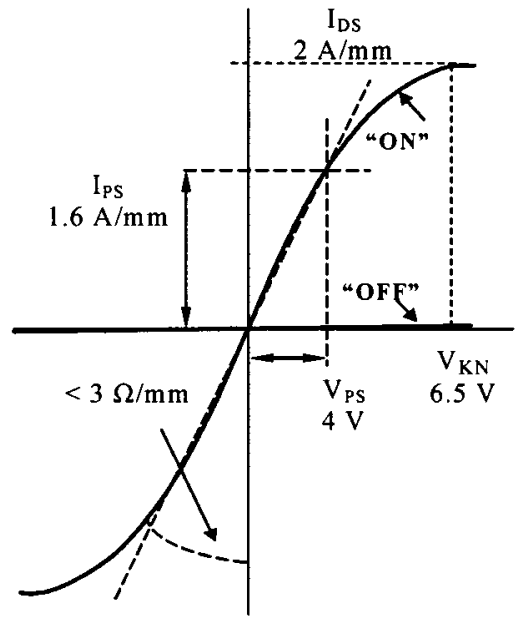

(b)

Fig. 3. (a) CW RF power sweep for 0.1-mm wide 1-A/mm MOSHFET switch and (b) the schematic $I-V$ characteristics of 2-A/mm device showing the mechanism of power saturation. Also shown are the peak voltage $V_{P S}$ and the current $I_{P S}$ corresponding to the switching power saturation.

device at saturation, $P_{m} \approx 150 \mathrm{~mW}$. This power corresponds to a peak voltage across the MOSHFET channel, $V_{P S} \approx 4.0 \mathrm{~V}$ and the peak current, $I_{P S} \approx 78 \mathrm{~mA}$. From the current-voltage $(I-V)$ characteristics shown in Fig. 3(b), we can see that $V_{P S} \approx 0.6 \mathrm{~V}_{K N}\left(I_{P S} \approx 0.78 I_{S M}\right)$. These data translate into the maximum switching power $P_{M O N} \approx 43 \mathrm{~W}$ for the 1-mm wide, $2-\mathrm{A} / \mathrm{mm}$ MOSHFET with the insertion loss, $L_{\text {Ins }}=0.27 \mathrm{~dB}\left(K_{p}=0.93\right)$. This power is more than $40 \times$ higher than that reported for the GaAs RF switches [3].

In the "OFF" state, the switching power $P_{M O F F}=$ $V_{B R}^{2} /\left(2 Z_{0}\right)$ is limited by the breakdown voltage $V_{B R}$ of the MOSHFET. As shown in [9], the $V_{B R}$ for the MOSHFET can be as high as $500 \mathrm{~V}$. "For the devices used for this experiment, the gate-drain spacing was not optimized for the highest $V_{B R}$ and $V_{B R}=175-220 \mathrm{~V}$. Taking the value of $V_{B R} \approx 200 \mathrm{~V}$, we estimate the maximum power to be $P_{M O F F} \approx 400 \mathrm{~W}$." This estimate shows that the "ON" state is the one that limits the high power performance of MOSHFET based RF switch.

\section{CONCLUSION}

The proposed novel AlGaN/GaN MOSHFET RF switch demonstrated low insertion loss of $0.27 \mathrm{~dB}$ and high insulation, about $40 \mathrm{~dB}$ at $100 \mathrm{MHz}$. The bandwidth of the switch (around $1 \mathrm{GHz}$ ) is limited by the wiring inductances in the ON state and by the device capacitance in the OFF state. The switch bandwidth can be significantly expanded by using the impedance matching circuits. The suggested switch features extremely high switching powers, about $43 \mathrm{~W}$ for $1-\mathrm{mm}$ wide device. These power levels are about $40 \times$ higher than those reported for GaAs FET switches.

\section{REFERENCES}

[1] A. Nagayam, M. Nishibe, T. Inaoka, and N. Mineshima, "Low-Iinsertion-loss DP3T MMIC switch for dual-band cellular phones," IEEE J. Solid-State Circuits, vol. 34, pp. 1051-1054, Aug. 1999.

[2] J. A. Torres and J. C. Freire, "Monolithic transistors SPST switch for L-band," IEEE Trans. Microwave Theory Tech., vol. 50, pp. 51-56, Jan. 2002.

[3] K. Miyatsuzi and D. Ueda, "A GaAs high power RF single pole dual throw switch IC for digital mobile Communication System," IEEE J. Solid-State Circuits, vol. 30, pp. 979-983, Sept. 1995.

[4] A. Gopinath and J. B. Rankin, "GaAs FET RF switches," IEEE Trans. Electron Devices, vol. ED-32, pp. 1272-1278, July 1989.

[5] S. Makioka, Y. Anda, K. Miyatsuji, and D. Ueda, "Super self-aligned GaAs RF switch IC with $0.25 \mathrm{~dB}$ extremely low insertion loss for mobile communication systems," IEEE Trans. Electron Devices, vol. 48, pp. 1510-1514, Aug. 2001.

[6] M. A. Khan, J. M. Van Hove, J. N. Kuznia, and D. T. Olsen, "High electron mobility GaN-AlGaN heterostructures grown by LPMOCVD," Appl. Phys. Lett., vol. 58, p. 2408, 1991.

[7] A. Koudymov, H. Fatima, G. Simin, J. Yang, M. A. Khan, A. Tarakji, X. $\mathrm{Hu}, \mathrm{M}$. S. Shur, and R. Gaska, "Maximum current in nitride-based heterostructure field effect transistors," Appl. Phys. Lett., to be published.

[8] M. A. Khan, X. Hu, G. Simin, J. Yang, R. Gaska, and M. S. Shur, "AlGaN/GaN metal-oxide-semiconductor heterostructure field effect transistors on SiC substrates," Appl. Phys. Lett., vol. 77, pp. 1339-1341, 2000 .

[9] G. Simin, X. Hu, N. Ilinskaya, A. Kumar, A. Koudymov, J. Zhang, M. A. Khan, R. Gaska, and M. S. Shur, "A $7.5 \mathrm{~kW} / \mathrm{mm}^{2}$ current switch using $\mathrm{AlGaN} / \mathrm{GaN}$ metal-oxide-semiconductor heterostructure field effect transistors on SiC Substrates," Electron. Lett., vol. 36, pp. 2043-2044, 2000

[10] G. Simin, X. Hu, N. Ilinskaya, J. Zhang, A. Tarakji, A. Kumar, M. A. Khan, R. Gaska, and M. S. Shur, "Large periphery high-power AlGaN/GaN metal-oxide-semiconductor heterostructure field effect transistors on $\mathrm{SiC}$ with oxide-bridging," IEEE Electron Device Lett., vol. 22, pp. 53-55, Feb. 2001.

[11] M. Ito and O. Wada, "Low-dark current GaAs MSM photodetector," IEEE J. Quantum Electron., vol. QE-22, p. 1073, 1986. 